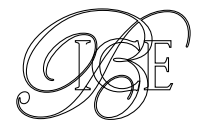

Inmaculada Gutiérrez Carrizo*

Marta Gómez Fernández**

\section{TODO ES MÁS GRANDE EN TEXAS}

El artículo analiza la economía tejana y las oportunidades que presenta para las empresas españolas. Con este fin se estudian variables tanto sociodemográficas como macro y microeconómicas, así como diferentes sectores. Además, se examinan las relaciones comerciales y los flujos de inversión entre este estado y terceros países, en especial con España, para el que Texas es el principal socio comercial dentro del conjunto de estados. Todo ello evidencia las grandes oportunidades de negocio que Texas ofrece. Por último, se han añadido los testimonios de empresas españolas de diversos sectores económicos que han desembarcado en Texas.

Palabras clave: economía tejana, flujos comerciales, inversión extranjera directa, oportunidades de negocio, sectores innovadores.

Clasificación JEL: F21, F23, J11, O51.

\section{Introducción}

Texas, con un PIB de 1.645 .135 millones de dólares en $2017^{1}$, es la segunda potencia económica de EE UU, tras California, y también el segundo estado más poblado, con 28,7 millones de habitantes censados. Es, además, después de Alaska, el estado más grande en extensión, con $695.660 \mathrm{~km}^{2}$, casi un $40 \%$ más que la superficie de España. A menudo se pone el ejemplo de que si fuera una nación independiente, estaría entre las doce mayores

\footnotetext{
* Consejera Económica y Comercial de España en Miami.

** Oficina Económica y Comercial de España en Miami. Versión de marzo de 2019.

DOI: https://doi.org/10.32796/bice.2019.3110.6791
}

1 Bureau of Economic Analysis, US Department of Commerce. economías del mundo, por encima de Rusia, Australia, España, Corea del Sur o México. Unas cincuenta compañías del 500 Fortune tienen su sede en Texas, el segundo estado del país con más corporaciones en este selecto club.

Pero Texas no solo resulta un mercado atractivo por su tamaño. También lo es por la diversificación sectorial que presenta, fundamentalmente hacia industrias y servicios de alto valor añadido: cuenta con recursos naturales (particularmente petróleo y gas), una industria manufacturera avanzada, fuerte presencia de sectores innovadores como el aeroespacial o la biotecnología y las ciencias de la vida, productos y servicios de alto valor añadido en el ámbito de las TIC, en particular en nichos tales como la ciberseguridad. Se trata de sectores dinámicos, con elevadas tasas de crecimiento y efecto arrastre sobre la economía. 
Esta diversificación también se produce en términos geográficos, porque las áreas que están protagonizando su crecimiento están diseminadas territorialmente a lo largo y ancho del estado. Texas cuenta con cuatro áreas metropolitanas con más de un millón de habitantes (Dallas-Fort Worth-Arlington, HoustonThe Woodlands-Sugar Land, San AntonioNew Braunfels y Austin-Round Rock). Además, tres de las diez ciudades que más crecen en Estados Unidos se encuentran en Texas (Houston, San Antonio y Dallas) ${ }^{2}$.

\section{Rasgos geopolíticos y sociodemográficos}

Texas, conocido en EE UU como el Estado de la Estrella Solitaria (Lone Star State), está

\footnotetext{
2 United States Census Bureau.
}

situado en el sur de Estados Unidos, bañado por el Golfo de México, con una frontera de casi $2.000 \mathrm{~km}$ con México, la mayor después de la frontera de Alaska y Canadá.

Desde un punto de vista demográfico, interesa resaltar, primero, su crecimiento. Según la Oficina del Censo de EE UU, en 2018 el estado de Texas tenía 28.701.845 habitantes, y aumentó un $1,4 \%$ respecto a 2017 y un $12,6 \%$ respecto a 2010. Estas cifras lo colocan como el estado con mayor crecimiento poblacional del país, que presentó en el mismo periodo un crecimiento medio del 5,5\%. De las 3.555.731 personas que se sumaron al censo entre abril de 2010 a julio 2018, 1.710 .031 fueron crecimiento poblacional natural de Texas, lo que lo sitúa como el segundo estado con un mayor crecimiento natural de población. Por otro lado, el saldo migratorio neto en este periodo de tiempo ha sido de 1.832 .775 habitantes, lo que convierte a Texas en el segundo estado con $\triangleright$

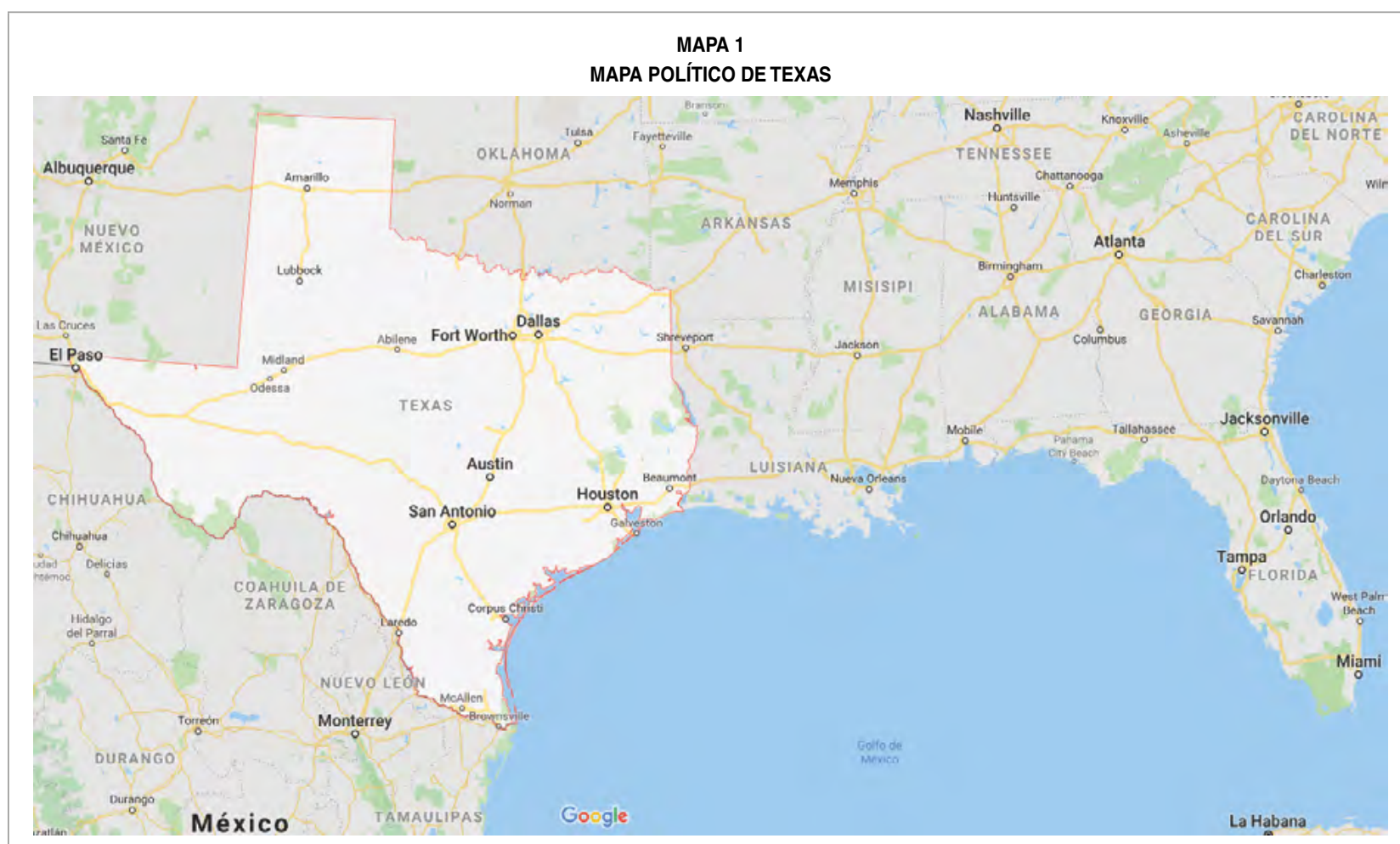

Fuente: Google Maps. 
TABLA 1

PRINCIPALES NÚCLEOS URBANOS DE TEXAS (ESTIMACIÓN 2017)

\begin{tabular}{|l|c|c|}
\hline \multicolumn{1}{|c|}{ Área metropolitana } & Número de habitantes & Variación 2013-2017 \\
\hline Houston-Sugar Land-Baytown & 6.636 .208 & $9,44 \%$ \\
San Antonio-New Braunfels & 2.377 .507 & $8,42 \%$ \\
Dallas-Fort Worth-Arlington & 7.104 .415 & $8,03 \%$ \\
Austin-Round Rock-San Marcos & 2.000 .590 & $12,26 \%$ \\
\hline Fuente: elaboración propia con datos del us Census Bureau. & \\
\hline
\end{tabular}

un mayor saldo migratorio, precedido solo por Florida. Debido a esta tendencia, se espera que la población en Texas alcance 35 millones de habitantes antes de 2040. Esto representaría aproximadamente un $68 \%$ de aumento de población con respecto a 2000. Por eso, Texas presenta una población comparativamente más joven que el resto del país. La media de edad en 2017 era de 34,3 años, el cuarto estado con la población más joven.

En segundo lugar, resulta destacable no solo el ritmo al que crece la población del estado, sino la diversidad de polos geográficos en la que lo hace. En Texas existen diversas grandes áreas metropolitanas, así como otras ciudades importantes, cuya conexión es una parte vital de la economía del país (Houston-Galveston, Dallas-Fort Worth, Austin —la capital del estado-, San Antonio, el Lower Rio Grande Valley, El Paso).

En tercer lugar, desde un punto de vista socioeconómico, es clave entender la composición de la población y quién está protagonizando este crecimiento. Según el último censo, en el periodo 2010-2017 la población hispanolatina aumentó en un $1,19 \%$, lo que la ha llevado de representar un $36,7 \%$ a un $38,9 \%$ del total de población; mientras que la población blanca no hispanolatina se redujo del $46,4 \%$ al $42,9 \%$. A su vez, se espera que la población hispana sobrepase a la anglosajona en 2022.

En Texas no existe lengua oficial; sin embargo, el inglés es la lengua más extendida. El español es también muy utilizado, con un $29,5 \%$ de población hispanoparlante. Este porcentaje se incrementa más en Dallas (37,9\%), Houston $(49 \%)$ o San Antonio $(39,7 \%)^{3}$.

\section{Cifras macroeconómicas}

Texas ha experimentado un crecimiento económico sobresaliente en los últimos años. Antes y después de la crisis exhibió tasas de crecimiento del PIB de en torno al 6-8\%, por encima de los principales estados del país. En 2017 el PIB de Texas fue un 39,5\% superior al de 2007, mientras que el PIB nacional creció un $33,9 \%$ en ese periodo 4 .

3 United States Census Bureau.

4 Bureau of Economic Analysis.

TABLA 2

DIFERENCIAS POBLACIONALES EN TEXAS, 2010-2017, Y SU CRECIMIENTO

\begin{tabular}{|l|c|c|c|c|c|}
\hline & $\mathbf{2 0 1 0}$ & $\begin{array}{c}\text { Porcentaje sobre } \\
\text { el total }\end{array}$ & $\mathbf{2 0 1 7}$ & $\begin{array}{c}\text { Porcentaje sobre } \\
\text { el total }\end{array}$ & $\begin{array}{c}\text { Tasa de crecimiento } \\
\mathbf{2 0 1 0 - 2 0 1 7}\end{array}$ \\
\hline Población total Texas & 24.311 .891 & 100,00 & 27.419 .612 & 100,00 & $1,12 \%$ \\
Población hispanolatina & 8.917 .477 & 36,70 & 10.673 .909 & 38,90 & $1,19 \%$ \\
Población no hispanolatina & 15.394 .414 & 63,30 & 16.745 .703 & 61,10 & $1,08 \%$ \\
\hline
\end{tabular}




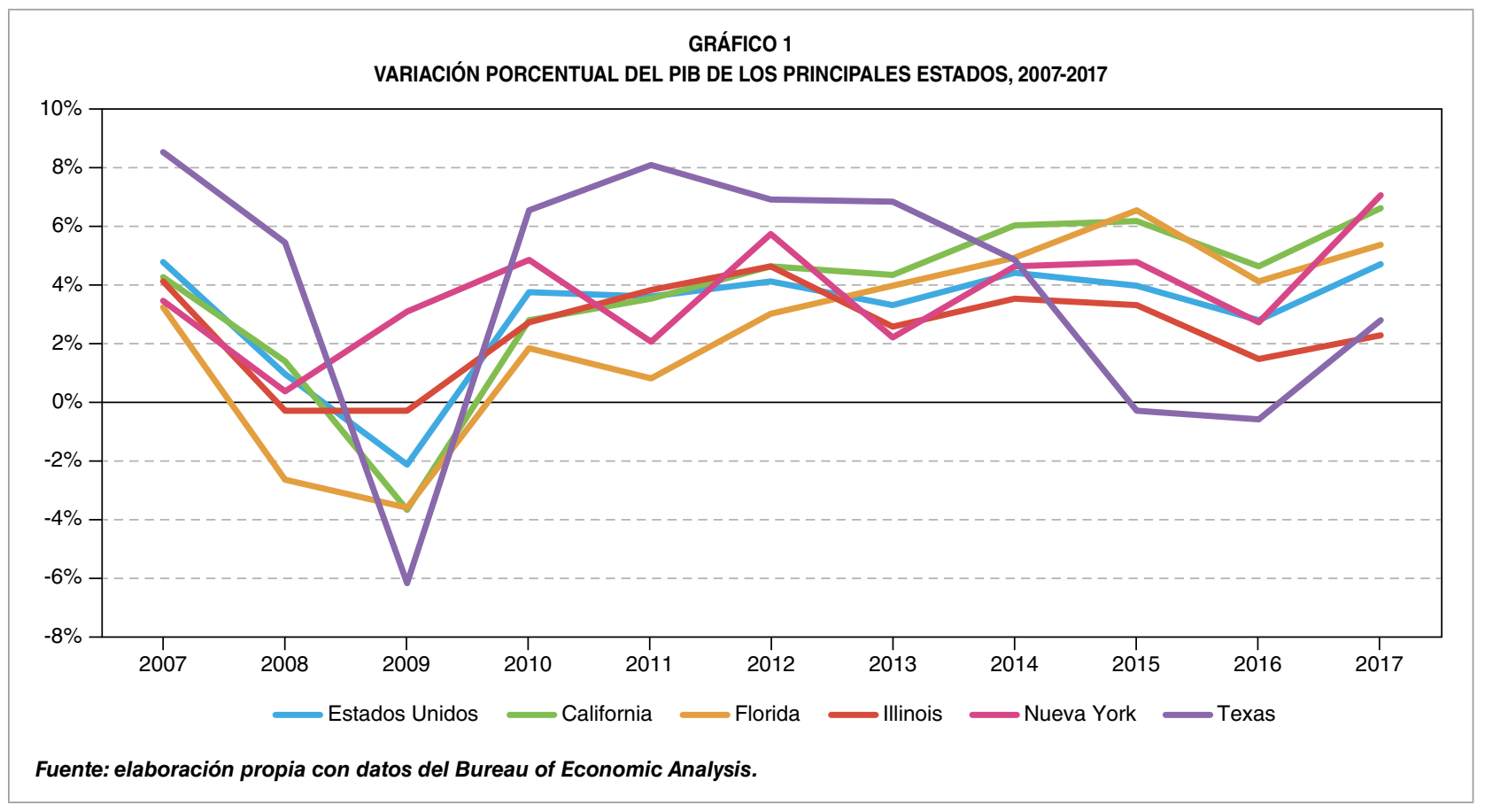

Resulta llamativo cómo, a finales de la década pasada, mientras la crisis económica sacudía fuertemente a otras economías occidentales y al resto del país, Texas tocó fondo y se recuperó con rapidez, a diferencia de lo que sucedió con otros estados como Florida, que se vieron mucho más afectados por la burbuja inmobiliaria y los problemas de solvencia de sus instituciones financieras.

El posterior ciclo de crecimiento en Texas se ha visto comprometido en los años 2015 y 2016 por la fuerte caída en los precios de petróleo y sus derivados, llegando a alcanzar tasas de crecimiento negativas en 2015 y 2016. Con todo, a diferencia de otras economías más dependientes de la industria del crudo y sus derivados, el grado de diversificación de la economía tejana ha protegido al estado de una crisis más aguda, y la recuperación económica ha sido mucho más rápida. Texas ha vuelto a recuperar la senda de crecimiento en 2017. De acuerdo con los datos de Texas Comptroller of Public Accounts, se espera que su PIB crezca de media un $2,6 \%$ en los próximos cinco años.
Este crecimiento económico viene posibilitando que el mercado de trabajo absorba el notable incremento de la población activa. Según el Bureau of Labor Statistics, Texas finalizó 2018 con una población activa de 13.902,5, de los cuales 13.387,4 eran población ocupada 5 . Esto sitúa la tasa de paro en un 3,7\%, por debajo de la media nacional.

Todo permite que el PIB per cápita de Texas no haya dejado de crecer en los últimos años. Según los últimos datos, esta variable ascendió a 57.077 en $2017^{6}$. Esto nos habla de un mercado atractivo por el poder adquisitivo de la masa de población que reúne.

\section{Estructura económica}

La economía de Texas presenta una sólida diversificación hacia sectores de alto valor añadido, con grandes perspectivas de crecimiento a futuro. Hacer aquí una descripción $\triangleright$

5 Datos todavía preliminares a febrero de 2019.

6 Bureau of Economic Analysis. 
pormenorizada de todo ello sería demasiado extenso. Nos ceñimos a aquellos ámbitos que las propias autoridades destacan como sectores de oportunidad para invertir en el estado.

\subsection{Energía}

Desde que en 1901 se descubriera el yacimiento de Spindletop, la economía de Texas ha estado profundamente ligada a este sector extractivo y a toda la industria de refino y petroquímica. Este hecho se ha visto reforzado a lo largo de los años por el hallazgo de sucesivos yacimientos de petróleo y gas. Texas es sede de más de cincuenta compañías del Fortune 1000 relacionadas con la industria del petróleo. Ello hace que hoy en día Texas sea el primer productor de energía del país en términos globales y que Houston sea conocida como la capital del mundo del petróleo, sede de más de 3.700 compañías y de un $40 \%$ de la capacidad petroquímica del país.

Pero, además, Texas ha sabido tradicionalmente diversificar su producción hacia otras fuentes (carbón, nuclear...) y, más recientemente, hacia las energías renovables. Hoy es el líder en generación de energía eólica, con más de 16,000 MW de capacidad instalados.

Por ello, Texas genera oportunidades de negocio para las empresas de la cadena de valor del oil\&gas (desde la exploración y la ingeniería hasta productores de válvulas y membranas), pero también en el ámbito de las energías renovables, donde las empresas españolas son líderes mundiales.

\subsection{Industria de tecnologías avanzadas}

Texas es un estado con una gran tradición de industria avanzada. La conjunción de un sistema educativo potente y grandes centros de investigación (como el Microelectronics Research Center de la Universidad de Texas, en Austin, o el Texas Center for Superconductivity de la Universidad de Houston), junto con un clima de negocios adecuado, ha hecho posible el desarrollo de una potente industria. En todo este fenómeno no hay que perder de vista que, históricamente, la existencia de abundantes recursos naturales (incluido, por supuesto, el petróleo) ha generado unos recursos que se han sabido canalizar adecuadamente para favorecer la diversificación económica hacia sectores de alto valor añadido. El liberalismo económico y la confianza en el mercado profundamente arraigados en el pueblo tejano han favorecido sin duda este proceso.

Hoy en día, dentro de la industria de las manufacturas operan 17.562 empresas, dando trabajo a más de 874.450 personas, con un salario medio de 71.500 dólares $(6,79 \%$ del total de población empleada). Destaca la presencia de empresas líderes globales como Texas Instruments, AMD, Samsung, Ericsson, Alcatel, Nokia, Fujitsu, Emerson, Toyota, General Motors o SMP. Se trata del estado que más manufacturas de alto valor añadido exporta, más de 20.000 millones de dólares en equipos de transporte.

\subsection{Aviación, aeroespacial y defensa}

Texas es sede de dos importantes aerolíneas internacionales (American Airlines y Southwest) y cuenta con dos de los aeropuertos con más tráfico del mundo: Houston y Dallas-Fort Worth, con más de 20 y 30 millones de pasajeros al año, respectivamente.

En Texas se encuentra el famoso Johnson's Space Center de la NASA, principal centro $\triangleright$ 
para misiones a la Estación Espacial Internacional. Ello hace de este estado, junto a Florida, uno de los epicentros del sector aeroespacial. La industria aeroespacial y de aviación emplea a 135.000 trabajadores a través de 1.300 empresas en el estado.

A ello se une la importancia que tiene el sector de defensa, que da empleo a unos 250.800 trabajadores entre militares en activo, Guardia Nacional y civiles trabajadores del Departamento de Defensa, con un impacto económico calculado en alrededor de $150.000 \mathrm{mi}-$ llones de dólares y un importante efecto arrastre sobre el resto de la economía.

\subsection{Biotecnología y ciencias de la vida}

Texas es la sede de más de cinco mil empresas que operan en el ámbito de la biotecnología y de las ciencias de la vida, que dan empleo directa e indirectamente a aproximadamente cien mil trabajadores. Empresas del Fortune 500 como Kimberly-Clark y Celanese tienen allí su base, además de contar con la presencia de líderes mundiales como Abbott, Allergan, Galderma, Johnson \& Johnson, McKesson o Novartis, entre otros.

Este estado alberga numerosas universidades de reconocido prestigio especializadas en el ámbito médico, seis de ellas en el top 100. A ello se une la presencia del Texas Medical Center de Houston, el mayor centro médico del mundo, que invierte más de 3.000 millones de dólares al año en I+D. Cuenta con 21 hospitales y 14 instituciones relacionadas, alberga a 50.000 estudiantes en diez instituciones académicas, 106.000 trabajadores y atiende a más de diez millones de pacientes al año?.

\subsection{Information \& Computer Tech}

En 1958, gracias al premio Nobel Dr. Kilby, vio la luz en Texas el circuito integrado que ha liderado la industria de los semiconductores, de la mano de la empresa Texas Instruments, la cual introdujo la calculadora diez años después. También parte de los inicios de la nanotecnología se encuentran en los estudios del Nobel Richard Smalley, profesor de la Universidad Rice de Houston. Dell Computers se fundó en Austin cuando Michael Dell era todavía un estudiante de la University of Texas.

Hoy en día Texas alberga más de 17.600 empresas de tecnología que emplean a más de 203.700 trabajadores, con un salario medio de 96.600 dólares. Se trata del segundo estado del país con más número de empresas especializadas en este sector, solo por detrás de California ${ }^{8}$.

El sector ha crecido significativamente en los últimos años gracias a los servicios online como los de almacenamiento en la nube, servicios de consultoría y ciberseguridad. San Antonio se ha consolidado como un centro de excelencia nacional para la ciberseguridad gracias al Lackland Air Force Base, que protege las operaciones de las Fuerzas Aéreas, y a la presencia del Centro de Criptología de la Agencia de Seguridad Nacional.

Otro subsector en relevancia es la industria de procesamiento de datos y almacenamiento. Texas da trabajo a 1 de cada 10 empleados del país en dichos servicios y acoge una de cada trece firmas. Destaca también la ciudad de Austin por la presencia de empresas especializadas en almacenamiento, procesamiento y gestión de datos.

8 Information \& Computer Technology Services Industry Report, 2018. 


\section{Apertura al exterior}

Texas es el estado que más exporta, por encima de California, posición que ha mantenido durante los últimos dieciséis años. En 2017 Texas exportó mercancías por valor de 264.541 millones de dólares, lo cual representa el 17,1\% del total de exportaciones estadounidenses de ese año. Entre las principales partidas de productos exportados se encuentran los electrónicos y ordenadores (17,8\% del total), los del carbón y petróleo $(16,7 \%)$, productos químicos $(15,3 \%)$ y petróleo y gas $(12,2 \%)^{9}$.

Sus principales socios comerciales son, con diferencia, México, con el que mantiene estrechas relaciones económicas y comerciales, puesto que muchas de las cadenas de producción están integradas con el país vecino, y, por detrás, Canadá. Destacan también por su volumen China, Brasil y Corea del Sur.

Como se observa en la Tabla 3 , tenemos que descender en el ranking a posiciones inferiores y cifras de exportación menos abultadas para encontrar socios comerciales europeos como destinos de las exportaciones tejanas. Desde un punto de vista agregado, las exportaciones de mercancías de Texas a la UE en 2017 no superaron el 12\% del total. Cifra similar alcanzan las importaciones tejanas de bienes procedentes de la UE. Con todo, Texas es el estado que más exporta a España, con 1.455.342.961 dólares en 2017, y constituye un importante socio comercial para nuestra economía, ya que importó mercancías españolas por valor de 1.841.627.551 dólares en 2017.

En cuanto a la composición de las exportaciones tejanas a España, lidera la partida de petróleo y gas $(36,3 \%)$, seguida de productos químicos (14,3\%), petróleo y productos derivados del carbón $(12,4 \%)$ y maquinaria no eléctrica $(10,2 \%)$. En el caso de España, Texas nos compra fundamentalmente maquinaria no eléctrica $(16,7 \%)$, seguido de productos químicos $(12,4 \%)$, productos minerales no metálicos $(12,1 \%)$ y manufactura de metales primarios $(11,9 \%)^{10}$.

Texas es una economía muy abierta a la inversión exterior. En 2016 fue el tercer estado que más inversión extranjera recibió, por detrás de California y Nueva York, aunque en determinados sectores, como el energético o el químico, lideró el ranking ${ }^{11}$. De hecho, Texas es el $\triangleright$

10 International Trade Administration.

11 Texas Economic Development and Tourism Division.

9 International Trade Administration, US Department of Commerce.

TABLA 3

PAÍSES DESTINO DE LAS EXPORTACIONES Y ORÍGENES DE LAS IMPORTACIONES DE TEXAS EN 2017 (En millones de dólares)

\begin{tabular}{|c|c|c|c|c|c|}
\hline \multicolumn{3}{|c|}{ Exportaciones } & \multicolumn{3}{|c|}{ Importaciones } \\
\hline Ranking & País & $\begin{array}{c}\text { Volumen de } \\
\text { exportaciones }\end{array}$ & Ranking & País & $\begin{array}{c}\text { Volumen de } \\
\text { importaciones }\end{array}$ \\
\hline 1 & México & 97.701 & 1 & México & 89.808 \\
\hline 2 & Canadá & 22.898 & 2 & China & 42.684 \\
\hline 3 & China & 16.419 & 3 & Canadá & 18.260 \\
\hline 4 & Brasil & 10.035 & 4 & Corea del Sur & 9.081 \\
\hline 5 & Corea del Sur & 9.723 & 5 & Arabia Saudí & 7.316 \\
\hline 6 & Japón & 8.863 & 6 & Alemania & 7.003 \\
\hline 7 & Países Bajos & 7.026 & 7 & Japón & 6.431 \\
\hline 8 & Singapur & 5.838 & 8 & Malasia & 5.601 \\
\hline
\end{tabular}


segundo estado con mayor volumen de empleo en empresas filiales de compañías extranjeras establecidas (585.900 empleos, con un 8,59\% del total de empleos generados por compañías extranjeras en EE UU), tras California (715.800 empleos, y un 10,49\% del total), así como el primero con mayor número de empleos creados por empresas extranjeras en el sector de servicios profesionales, técnicos y científicos y el segundo en el sector industrial ${ }^{12}$.

Texas Economic Development, dependiente del gobernador, ofrece datos detallados sobre la inversión extranjera en Texas. Entre enero de 2011 y marzo de $2016^{13}$ se registraron 1.441 proyectos de inversión directa extranjera, de los cuales 761 fueron proyectos greenfield, que representaron una inversión total combinada de 212.000 millones de dólares. En este caso el panorama es diferente al que observamos a la hora de analizar los principales socios comerciales. Si en el caso de las exportaciones e importaciones de bienes, México, Canadá y determinados países asiáticos son los protagonistas, en el caso de la inversión directa, más del 50\% proviene del oeste de Europa.

A su vez, Texas invirtió en el extranjero en ese período 59.166 millones de dólares en 847 proyectos por parte de 433 empresas y creó alrededor del mundo 95.574 puestos de trabajo. Es el estado que más invierte en el extranjero en proyectos de energía e industria química. Los principales destinos de la inversión tejana en el resto del mundo, entre enero de 2011 y marzo de 2016, fueron: Reino Unido (con 106 proyectos y 1.631 millones de dólares), China (con 67 proyectos y 2.063 millones de dólares), India (con 60 proyectos y 3.189 millones de dólares), Canadá (con 53 proyectos y $11.125 \mathrm{mi}-$ llones de dólares) y México (con 39 proyectos y 5.277 millones de dólares) ${ }^{14}$.

\section{Inversión española en el estado}

En los últimos años la inversión española en Texas no ha dejado de crecer. De acuerdo con los datos de ICEX, el número de empresas españolas implantadas y con presencia en Texas es de al menos 100, siendo la mayoría grandes empresas o medianas-grandes y habiéndose instalado o reforzado la presencia de muchas de ellas en el último decenio.

Los datos de Texas Economic Development sitúan a España entre 2011 y 2016 como el séptimo mayor inversor en Texas, con 27 proyectos y 835,3 millones de dólares. Según esta misma fuente, 23 empresas inversoras $\square$

12 Bureau of Economic Analysis

13 Texas Economic Development and Tourism Division.

14 Texas Economic Development and Tourism Division.

TABLA 4

INVERSIÓN EN TEXAS SEGÚN PAÍS

\begin{tabular}{|l|c|c|c|c|}
\hline \multicolumn{1}{|c|}{ País } & N. $^{\circ}$ proyectos & Capex (en millones) & Puestos de trabajo & N. $^{\circ}$ compañías \\
\hline Reino Unido & 165 & $2.296,50$ & 6.136 & 146 \\
Alemania & 80 & $3.035,90$ & 7.521 & 63 \\
Canadá & 77 & $2.731,50$ & 9.219 & 72 \\
Francia & 54 & $4.464,40$ & 5.664 & 45 \\
Japón & 49 & $3.098,80$ & 12.354 & 44 \\
Suiza & 29 & 868,40 & 3.577 & 25 \\
España & 27 & 835,30 & 3.462 & 23 \\
\hline \multicolumn{2}{l|}{} \\
\hline
\end{tabular}


españolas crearon 3.462 empleos, principalmente en los sectores de finanzas, servicios profesionales, tecnologías de la información, electrónica, energías renovables y bienes de consumo industrial.

Hacer una relación pormenorizada de estas inversiones sería extenso. Pero destacamos a continuación algunos ejemplos significativos. Así, el Grupo Ferrovial, que gestiona en Texas una inversión total de 6.300 millones de dólares, ha localizado en la capital, Austin, la sede para EE UU de varias de sus sociedades (Ferrovial Agroman, Cintra, Ferrovial Servicios, entre ellas). En Texas también se localizan las sedes de otras ingenierías y empresas de servicios españolas, como Grupo Álava, Acciona Infraestructuras, Iberdrola, OHL Industrial, o Prointec, entre otras. Se trata de un estado al que, en general, apuntan todas las grandes empresas de infraestructuras y servicios de ingeniería españoles.

Esta llegada de empresas está llamada a intensificarse. Recientemente se ha sabido que Texas Central Partners, promotor privado del proyecto de alta velocidad entre Houston y Dallas, ha elegido a Renfe y a Adif como socios estratégicos. Tras la primera fase como asesores técnicos en el desarrollo, diseño y construcción, Texas Central prevé que Renfe y Adif asistan en los planes posteriores de operación y mantenimiento para la puesta a punto del servicio, incluyendo la operación de los trenes, el mantenimiento de los equipos y otros servicios relacionados con la comercialización de billetes.

Repsol cuenta con presencia en EE UU a través de su filial Repsol Energy North America, situada en el estado de Texas, con más de 400 empleados. La empresa tiene una importante presencia en la zona del Golfo de México estadounidense desde 2003, una de las zonas de aguas profundas de mayor rentabilidad de la industria petrolera mundial.
La presencia española es también destacable en el ámbito de las energías renovables. Iberdrola (Avangrid Renewables) está presente en el estado con importantes plantas de generación eólica en el norte (Barton Chapel) y sureste del estado (Peñascal I y II y Baffin). Texas es también un estado estratégico para Acciona Energía, donde la empresa cuenta con los parques eólicos de San Román y de Palmas Altas.

Hay que destacar también el impresionante desarrollo del negocio de FFC Environmental en los últimos años. La empresa ha conseguido contratos millonarios para la gestión y reciclaje de los residuos sólidos de diferentes ciudades, incluidas Mesquite, Garland (de las ciudades más grandes en Dallas-Fort Worth Metroplex) y, recientemente, Houston, donde está construyendo una moderna planta de reciclado.

Las empresas españolas están también presentes en sectores industriales, como en la fabricación de embalajes de plástico (SPR Packaging), de munición (Rio Ammunition), ejecución de proyectos industriales (Control y Montaje CYMI), electrodomésticos industriales (Fagor), manufactura de cadenas (Vicinay International Chain), etcétera.

El sector de servicios financieros ha sido el que tradicionalmente ha acumulado una mayor inversión. En Texas destaca la presencia de BBVA, a través de su filial BBVA Compass. Tras importantes operaciones de compra (incluido Compass Bank en 2007), BBVA Compass se ha convertido en uno de los 25 bancos comerciales más importantes de EE UU por cuota de mercado de depósitos. Es considerado el 2.ำ banco en Alabama, el 4. en Texas y el 5. en Arizona. Concretamente, en Texas es donde tiene una mayor red de sucursales, 344 de las 672 que tiene en el país, e importantes oficinas corporativas. Grupo Santander tiene también una importante presencia en el estado, tanto $\triangle$ 
en banca de inversión como con su filial de crédito al consumo, Santander Consumer Bank.

El sector alimentario también cuenta con presencia significativa de la empresa española. La multinacional española con sede en Houston, Ebro Riviana, es el primer productor de arroz del país y líder también en el sector de la pasta. Más recientemente ha aterrizado, en San Antonio, Cerealto, filial del grupo SIRO que comercializa pasta, galletas, cereales o snacks.

En Texas tienen también importante presencia empresas españolas líderes en diferentes segmentos del sector del hábitat, como Cosentino o Roca, o en la comercialización de piedra, como Levantina.

Como se ha señalado anteriormente, son más de un centenar los grupos españoles que han decidido establecerse y crecer en territorio tejano. La enumeración de proyectos aquí realizada no deja de ser incompleta y, por tanto, no hace verdadera justicia al esfuerzo inversor español en ese estado.

\section{Oportunidades y retos para la empresa española}

Diversos factores hacen de Texas un mercado atractivo para la empresa española.

\subsection{Carácter geoestratégico}

Como hemos analizado, Texas es un mercado con un gran tamaño, en crecimiento, localizado geográficamente en una zona que permite un fácil acceso al resto del mercado norteamericano, con una especial vinculación $\triangleright$

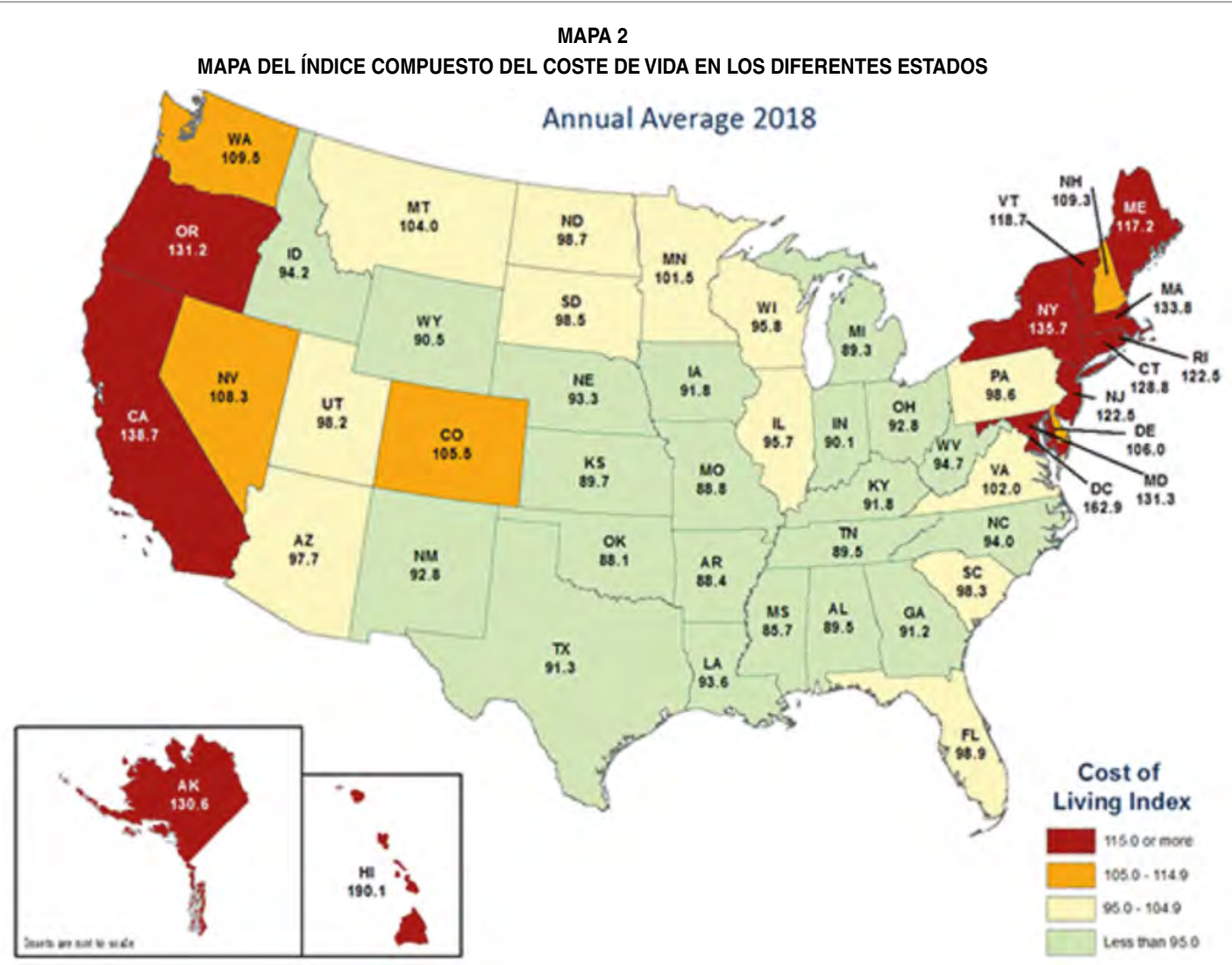

Fuente: Missouri Department of Economic Development. 
con México, donde la empresa española tiene una considerable penetración. Por otro lado, Texas ofrece a nuestras empresas una cercanía cultural mucho mayor que la de otros estados. No hay que olvidar la presencia española que históricamente se produjo en Texas (especialmente ostensible en zonas como San Antonio) y la influencia hispana, cada vez más patente gracias al incesante impulso de la inmigración.

\subsection{Coste de la vida comparativamente bajo}

Los precios del suelo y la vivienda, los productos básicos y los servicios varían de manera muy significativa de unas zonas a otras del país. Comparado con otros de los principales estados, los costes en Texas resultan relativamente baratos. Desde 1968 The Council for Community and Economic Research (C2ER) viene realizando una encuesta entre más de 300 áreas urbanas y elaborando trimestralmente un índice del coste de la vida denominado C2ER Cost of Living Index (COLI). Las autoridades de desarrollo de diferentes estados observan a menudo este índice para valorar su posición competitiva ${ }^{15}$.

15 Ver, por ejemplo, el análisis del Missouri Department of Economic Development.
Texas es, con mucha diferencia, el estado con menor coste de vida de los principales estados del país. La diferencia es especialmente llamativa cuando se compara con California o Nueva York (Tabla 5).

Esta comparativa resulta especialmente relevante si, además del coste de la vida, tenemos en cuenta el PIB per cápita de cada estado.

En el Gráfico 2 se observa precisamente cómo se posicionan los diferentes estados en cuanto a PIB per cápita y a coste de la vida. Así, vemos cómo Texas tiene un PIB per cápita relativamente alto (57.077 dólares) y, a la vez, un bajo índice de coste de vida $(91,3$, siendo 100 la media), lo que lo coloca en una posición muy competitiva.

\subsection{Baja presión fiscal}

Texas es uno de los estados fiscalmente más ventajosos para las empresas y las personas físicas. Es uno de los siete estados que no aplica impuesto de sociedades a escala estatal (tampoco aplica un impuesto sobre la renta de las personas físicas). Además, no permite aplicarlo a otras subdivisiones ejecutivas (condados o ciudades). Sí impone un impuesto sobre los ingresos llamado Franchise Tax o Margin Tax, cuyo tipo impositivo es del $0,750 \%$ para la mayoría de las entidades y menor para $\triangle$

TABLA 5

MEDIA DEL COSTE DE VIDA ANUAL EN LOS PRINCIPALES ESTADOS (DIVERSAS VARIABLES)

\begin{tabular}{|l|c|c|c|c|c|c|r|r|}
\hline \multicolumn{10}{|c|}{ Mstado } & Ranking & Índice & Compra & Vivienda & Transporte & Servicios públicos & Sanidad & Otros \\
\hline California & 49 & 138,7 & 112,2 & 196,1 & 122,0 & 121,6 & 110,8 & 116,6 \\
Florida & 30 & 98,9 & 98,2 & 96,1 & 99,2 & 101,3 & 95,5 & 106,2 \\
Illinois & 22 & 95,7 & 98,6 & 88,2 & 104,2 & 96,7 & 100,3 & 97,0 \\
Nueva York & 48 & 135,7 & 112,7 & 197,1 & 107,9 & 105,5 & 103,9 & 110,6 \\
Texas & 12 & 91,3 & 94,0 & 84,3 & 94,4 & 101,0 & 95,2 & 89,9 \\
\hline
\end{tabular}

Fuente: elaboración propia con datos del Missouri Department of Economic Development. 


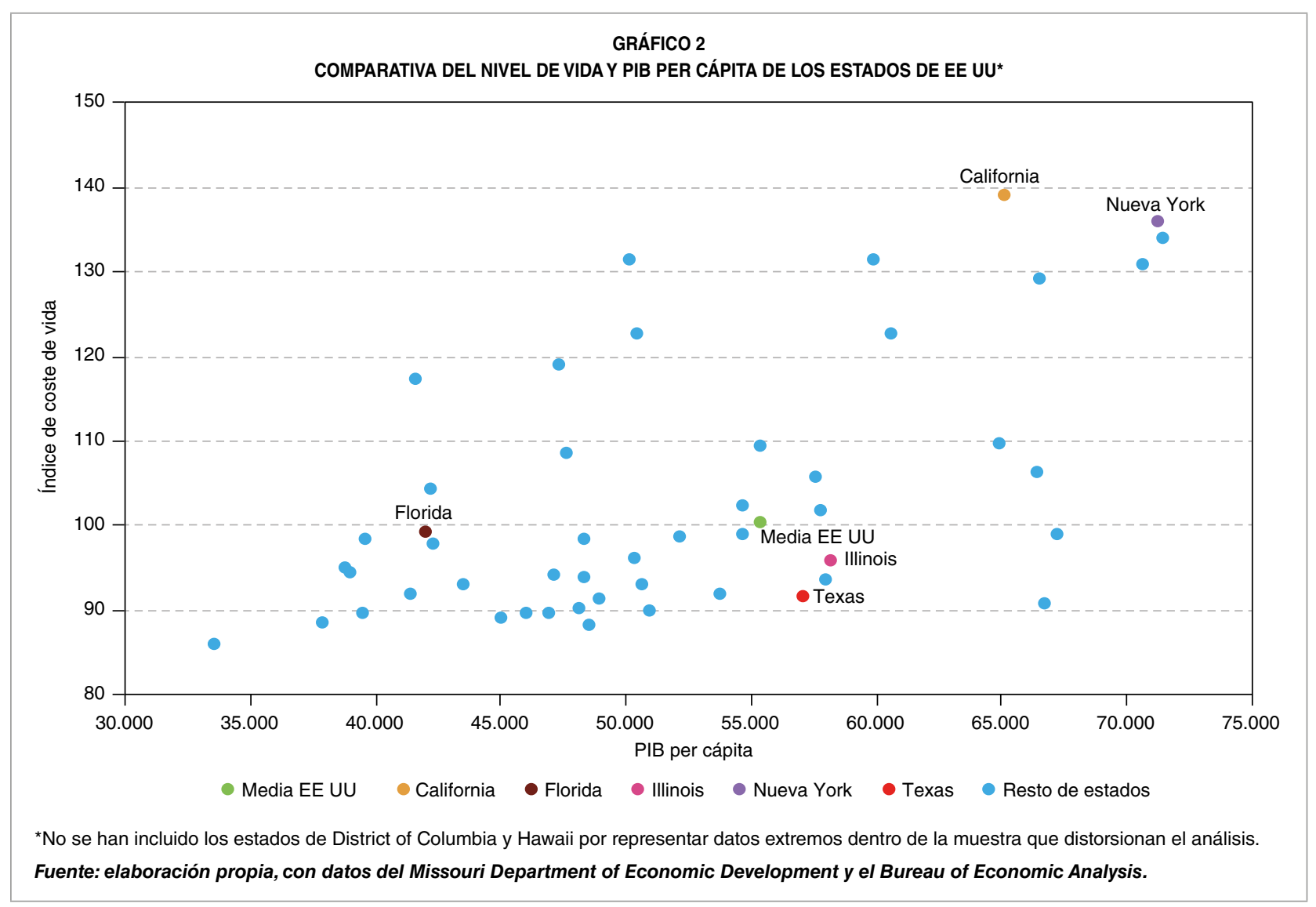

determinados distribuidores mayoristas y minoristas y para entidades con unos ingresos de 20 millones de dólares o menos.

La baja presión fiscal, el relativamente bajo coste de la vida, el dinamismo de su economía y las posibilidades que su mercado de trabajo ofrece, está provocando en los últimos años que no pocos profesionales y empresas del país se muden a Texas.

\subsection{Clima de negocios}

Tanto a nivel estatal como local, las autoridades del estado llevan a gala su afán por favorecer un mercado donde la Administración ocupe el menor espacio posible y la regulación no obstaculice el crecimiento de los negocios. La explicación, a este respecto, del órgano de desarrollo económico del estado, la Texas Economic Development Corporation, es muy elocuente ${ }^{16}$ :

«Texas offers companies and entrepreneurs room to grow, as well as the freedom to go big and to be rewarded for their ambitions. Less government, lower taxes, smarter regulations and right-to-work laws are the economic policies attracting employers to Texas from states that overtax and over-regulate».

Los hechos, efectivamente, atestiguan que esa es la inspiración de las autoridades del estado al regular. Como se ha visto, Texas no tiene impuesto sobre las personas físicas o jurídicas. Asimismo, es uno de los 27 estados $D$

16 https://businessintexas.com/why-texas/fair-legal-system 
que ha aprobado «leyes de derecho al trabajo» (right-to-work laws). Esta normativa garantiza que a ninguna persona se le pueda obligar, como una condición de empleo, a unirse o a no unirse, ni a pagar cuotas a un sindicato. El fundamento de estas normas no es tanto una oposición al movimiento sindical como la creencia en la libertad individual, profundamente arraigada en la sociedad tejana. Esta desindicalización de las relaciones laborales favorece una mayor flexibilidad del mercado laboral.

\subsection{Retos para la empresa española}

Sin duda, el potencial económico y el clima de negocios de este estado resultan atractivos. Con todo, la penetración en un mercado como el tejano no está exenta de dificultades.

Quien quiera hacer negocios en este territorio debe tener muy presente que debe adecuarse a la cultura y exigencias locales. El individuo tejano es prototípicamente bravo y está intrínsecamente convencido de su capacidad y valor («The land of the free and the home of the brave...» reza el estribillo de uno de sus himnos nacionales). Esta idiosincrasia tejana debe ser tenida en cuenta a la hora de hacer negocios. Es necesario presentar las propuestas con solidez, rigor y seguridad, reconociendo y conociendo la potencia de ese mercado. Hay que ganarse el respeto de los tejanos, que, por otra parte, constituyen una colectividad que acoge con cariño y respeto a aquellos que llegan a su suelo con ganas de trabajar duro.

El hecho de que el inglés no se haya declarado idioma oficial (de nuevo, la libertad individual...) y de que la presencia hispana sea extensa no debe engañarnos. El idioma de los negocios es el inglés. No solo eso. Como a menudo sucede, conviene involucrar de una forma o de otra a locales en el proyecto para que la inmersión en el mercado sea verdaderamente efectiva.

Cualquier proyecto de implantación en el mercado va a requerir contar con capacidad financiera sólida. El hecho de que el coste de vida sea relativamente bajo en relación a otros estados potentes del país no evita que el desarrollo de un proyecto en Texas requiera de recursos que permitan afrontar el reto de desarrollar un proyecto en un mercado exigente.

A menudo las empresas españolas implantadas en Texas - y no solo en Texas- consideran que uno de los principales escollos a la hora de desarrollar sus proyectos es contar con los profesionales adecuados a un coste «razonable». En primer lugar, la diferencia en el nivel de los salarios del mercado español con el americano suele ser un choque «cultural» para muchas empresas españolas, además de económico. Por otra parte, la flexibilidad del mercado laboral es sin duda atractiva, pero lleva también a que la rotación en los empleos sea mayor y a que los trabajadores sean mas propensos a cambiar de empleo ante ligeras mejoras de las condiciones. Esto, en un mercado cercano al pleno empleo y una demanda pujante, hace que captar talento y profesionales cualificados sea más difícil.

En definitiva, Texas es una economía en crecimiento, que atrae, por su potencial, proyectos de inversión. Haciendo una propuesta de valor convincente respaldada por los recursos suficientes surgirán las oportunidades de negocio. Este puede ser un hecho común a otros estados, pero, en comparación con ellos, la empresa española puede encontrar en Texas $y$, en general, en el sudeste del país, un clima de negocios más propicio. Ya lo dice el popular dicho tejano, atribuido a Davy Crockett: «Y'all can go to hell... I'm a-goin' to Texas». 


\section{ANEXO \\ CONTRIBUCIÓN DE EMPRESAS ESPAÑOLAS EN TEXAS}

\section{EBRO RIVIANA}

\section{¿Cuál es la presencia de su empresa en Estados Unidos y, en particular, en Texas?}

Nuestra entrada en Estados Unidos se remonta a 2004, año en que adquirimos Riviana Foods, empresa líder de arroz en EE UU. Su incorporación permitió al Grupo Ebro entrar en uno de los mayores mercados del sector de la alimentación y, al mismo tiempo, disponer de una potente red de distribución, ya que Riviana atesoraba una importante posición de liderazgo en 19 de los 20 principales estados más importantes de EE UU.

Asimismo, la incorporación de la compañía norteamericana consolidó a Ebro como el primer grupo arrocero del mundo, con un posicionamiento líder tanto en Europa como en Estados Unidos.

Años más tarde, en 2011, el Grupo incorporó al perímetro arrocero la empresa American Rice (ARI), ubicada en Freeport (Texas). Y cuatro años después, en 2015, adquirimos, también allí en Texas, RiceSelect, negocio líder en el mercado estadounidense de arroces aromáticos y orgánicos y de productos basados en ancient grains, quinua y otros supercereales. Un complemento perfecto para el portfolio de Riviana e idóneo para reforzar su desarrollo en los segmentos de mayor crecimiento del mercado norteamericano de arroz.

Con esta operación, Ebro finalizó (por el momento) su plan de expansión internacional en USA, que pasó a convertirse en el principal mercado geográfico del Grupo.

Hoy por hoy, Ebro dispone de una amplia presencia en Estados Unidos. Nuestras oficinas centrales se ubican en Houston y tenemos un amplio parque industrial, con 12 plantas productivas situadas en Memphis, Freeport, Carlisle, Brinkley, Hazen, Crowley, Alvin y Clearbrook para el Área de Arroz; y Saint Louis, Winchester, Fresno, Memphis y Hamilton para la División de Pasta. Para la modernización de dicho parque industrial hemos invertido cerca de 294 millones de dólares en los últimos diez años. En Houston también se ha instalado el equipo global de IT del Grupo Ebro; desde allí, este equipo de profesionales, formado por una plantilla fundamentalmente española, da servicio a toda la compañía en sus distintas ubicaciones en el mundo.

\section{¿Por qué eligieron Texas como sede para el desarrollo de sus operaciones?}

Siendo sinceros, nuestra elección fue Riviana, su adquisición fue lo que determinó nuestra presencia en Texas, si bien es cierto también que después, como señalado anteriormente, hicimos más inversiones en este estado, por ser una de las principales zonas productoras de arroz largo, junto a Arkansas y Luisiana.

\section{¿Qué ventajas ofrece Texas frente a otros estados?}

Es uno de los estados con mayor población de Estados Unidos, lo que ofrece importantes perspectivas tanto de desarrollo como de crecimiento para todo tipo de negocios; tiene una economía sólida y diversificada y una excelente ubicación para la exportación. Es también un estado que ofrece acceso a una importante masa de mano de obra cualificada, buena logística de entrada al país y un perfil hispano que proporciona buena conectividad con México/Centroamérica.

¿Cuáles son las mayores dificultades o retos a los que se ha enfrentado su empresa y en qué medida son particulares de este estado?

El mayor reto lo estamos afrontando ahora como consecuencia de la situación de pleno empleo que hay en Estados Unidos y que concretamente a nosotros nos afecta de modo especial en nuestra fábrica de Freeport. Estamos encontrando serias dificultades para encontrar mano de obra cualificada y haciendo un gran esfuerzo económico para retener el talento, ya que los trabajadores están decantándose por la industria petroquímica en detrimento del resto de sectores.

\section{¿Qué oportunidades ven en Texas a futuro?}

Una serie de factores, como la perfecta unión que ha habido entre el Grupo Ebro y Riviana, el amplio knowhow que hemos adquirido del mercado estadounidense gracias a todos estos años de experiencia y las $\square$ 
inmensas oportunidades que ofrece un país como Estados Unidos, que están propiciando que nuestra aventura norteamericana esté siendo muy satisfactoria, que nos sintamos muy confortables, y, por tanto, si surgen oportunidades, seguiremos creciendo.

¿Qué recomendarían a la empresa española que mira a Texas como mercado para su expansión?

Dependiendo del tipo de negocio de cada empresa, es crucial dedicar tiempo a visitar el mercado y entender las diferencias entre las diferentes zonas (Houston, Dallas, San Antonio, etcétera). Texas tiene suficiente masa crítica e internacionalidad como para ofrecer socios/clientes que pueden servir de cabeza de playa para desarrollar el mercado americano o crear una presencia física en Estados Unidos. Es importante dedicar tiempo a entender quién puede ser ese compañero de viaje. Así, por ejemplo, para las empresas alimentarias la cadena de supermercados texana HEB es un excelente punto de llegada y una manera de ganar experiencia en un mercado tan exigente como el americano.

\section{GRUPO ÁLAVA}

\section{¿Cuál es la presencia de su empresa en Estados Unidos y, en particular, en Texas?}

Aterrizamos en Estados Unidos en marzo de 2014, así que acabamos de cumplir cinco años instalados aquí. Desde el primer momento lo tuvimos claro: vinimos con intención de quedarnos y nuestro objetivo es crecer en el mercado americano y convertirnos en una empresa multinacional con una importante base en Norteamérica desde donde liderar nuestra actividad en el continente americano.

En nuestro caso, la pregunta no puede ser más oportuna, porque precisamente fue Texas nuestra puerta de entrada a Estados Unidos, como creo que también lo ha sido de muchas otras empresas españolas.

Comenzamos aquí nuestra andadura instalando una oficina muy pequeña en Fort Worth mientras aterrizábamos y aprendíamos a conocer mejor cómo funcionaba este mercado. Afortunadamente, pronto conseguimos hacernos un hueco en el mercado texano de servicios de ingeniería civil y ganamos varios contratos, tuvimos que aumentar nuestra plantilla y esta oficina se nos quedó pequeña. Llegó un momento en el que tuvimos que plantear un cambio de sede y decidimos ampliar nuestras instalaciones y mudarnos a la ciudad de Dallas, ya que allí se encontraban algunos de nuestros nuevos colaboradores.

En la actualidad hemos rediseñado nuestra estrategia en el país y en los próximos meses abriremos dos nuevas oficinas, una en Los Ángeles y otra en Miami, para atender mejor y de manera más cercana toda nuestra actividad comercial, que se distribuye en tres grandes áreas geográficas de Estados Unidos: Costa Oeste, Centro Sureste y Costa Este.

Tenemos claro que Estados Unidos es un mercado muy interesante y puede ofrecer muchísimas oportunidades para una empresa como la nuestra, dedicada a servicios y soluciones para grandes proyectos de infraestructuras. Pero si algo hemos aprendido en estos cinco años de experiencia es que también es uno de los mercados más competitivos del mundo: aquí están instaladas las mejores empresas y la competencia es feroz. Si quieres crecer y no ser solo una estrella fugaz, tienes que hacer una apuesta muy fuerte por diferenciarte y ser el mejor en lo que haces.

\section{¿Por qué eligieron Texas como sede para el desarrollo de sus operaciones?}

Como he comentado anteriormente, Texas ha sido nuestra puerta de entrada a Estados Unidos. Cuando decidimos dar el salto vimos que Texas reunía una serie de características óptimas para nuestra implantación en el país: es uno de los principales mercados americanos, está muy abierto a la inversión extranjera, tiene un régimen fiscal más «amigable» y además se encuentra en un proceso importante de construcción y reconstrucción de grandes infraestructuras de transportes, lo que hace que sea un mercado ideal para la implantación de una empresa como Álava International. 
Este último aspecto es fundamental. Creo que Texas es uno de los estados que está apostando más fuerte por crear una red de infraestructuras de primer nivel capaz de conectar las principales ciudades y servir de corredor entre los estados del sur. El potencial de Texas en este sentido es impresionante, como lo es el de algunas ciudades, como el área metropolitana Dallas-Fort Worth, Houston, Austin o San Antonio.

\section{¿Qué ventajas ofrece Texas frente a otros estados?}

Una de las ventajas principales, que a nadie se le puede pasar por alto, es que es uno de los estados que ofrece un acceso más fácil al mercado norteamericano para una empresa extranjera. Es algo evidente y además necesario.

Texas posee uno de los mejores regímenes fiscales del país y permite operar realizando menos trámites legales o administrativos si comparamos con las exigencias burocráticas de otros estados. Nuestra experiencia además es que aquí se valora mucho la experiencia y la solidez financiera que puedas ofrecer a tus clientes, y esto es fundamental para que el desarrollo comercial los primeros meses sea mucho más ágil.

Otro punto importante es que está perfectamente conectada con mercados muy interesantes como California o Florida, tanto por avión como por carretera, y está sabiendo capitalizar como ningún otro estado el potencial y las oportunidades que ofrece el sur del país.

En la última década el estado de Texas ha sabido modernizar y desarrollar su enconía realizando una fuerte inversión en infraestructuras y apoyando la creación de nuevas empresas en su territorio. Aunque siga teniendo un peso importante en su economía la ganadería, con sus famosos cowboys y ranchos, y el mercado del oil\&gas, la apuesta por las nuevas tecnologías y el empeño en convertirse en un estado puntero está consiguiendo que su economía crezca de forma imparable cada año, y que sea una apuesta segura para la inversión de compañías extranjeras y nacionales.

Podemos decir que es un estado "en fase de expansión y reconstrucción», ya que ha invertido mucho dinero en infraestructuras para conectar grandes centros poblacionales, universitarios, tecnológicos, empresariales, logísticos y residenciales. Un ejemplo evidente es que muchas empresas - tanto norteamericanas como internacionales- están abriendo nuevas sedes en Texas, lo que a nosotros nos permite tener un networking como empresa cada vez de mayor nivel.

\section{¿Cuáles son las mayores dificultades o retos a los que se ha enfrentado su empresa y en qué medida son particulares de este estado?}

Los principales problemas que nos hemos encontrado, creo, tienen más que ver con nuestro propio modelo de negocio que con las peculiaridades que pueda tener un determinado estado. Estoy convencido de que todas las empresas que decidimos aterrizar en un nuevo mercado internacional nos encontramos con una serie de obstáculos que vas superando día a día y paso a paso; lo importante es ir aprendiendo en el proceso. A día de hoy no conozco ninguna empresa que haya tenido un camino limpio y sin obstáculos. Es parte del juego, y es bonito también ir superando retos y obstáculos.

En nuestro caso, aprendimos muy rápido que era muy importante, y diría que prácticamente fundamental, cambiar la mentalidad con la que trabajábamos en Europa. Estábamos acostumbrados a iniciar toda la maquinaria de ventas y de gestión después de obtener un contrato o un pedido, y aquí eso «no vale»; has de demostrar confianza y apuesta por el mercado en el que te mueves, invirtiendo y desarrollando actividad, para que tus clientes potenciales confíen en ti.

Afortunadamente, el grupo empresarial cuenta con un buen pulso financiero, y pudimos invertir en preparar proyectos de una facturación importante que no todas las empresas de nuestro tamaño pueden permitirse.

A nivel económico creo que es importante tener claro que debes realizar un gran desembolso económico para iniciar actividades comerciales, sobre todo cuando no tienes un histórico contrastable en el país y en el mercado, como el alto coste de seguros, abogados, gastos bancarios para acceder a créditos, o accesos a leasing, por ejemplo. 
En cuanto a visados y cuestiones migratorias, después de cinco años realizando las cosas de forma metódica y cumpliendo exactamente con las directrices legislativas, nuestra compañía ha conseguido el certificado y reconocimiento como E2 Treaty Company, que es algo complicado de obtener, y esto nos facilita mucho los trámites administrativos en la embajada, que al principio pueden resultar muy arduos.

\section{¿Qué oportunidades ven en Texas a futuro?}

Es indiscutible que Texas no es solo una apuesta de presente para Álava International, sino que también es una apuesta de futuro. Nadie puede negar el potencial que tiene este mercado y el esfuerzo que está haciendo para igualarse a los estados más punteros de Estados Unidos; es uno de los estados más dinámicos del país.

Si bien es cierto que entramos en el mercado centrándonos en nuestros servicios para la obra civil, que es un nicho de mercado que conocemos a la perfección, nuestro plan de desarrollo de negocio de la compañía pasa por desarrollar en este mercado nuestras áreas de negocio de soluciones tecnológicas de alto nivel. Cada vez vemos más claras las oportunidades para diversificar nuestro portfolio hacia este tipo de soluciones, como ya hacemos en España.

Vemos que cada vez un mayor número de empresas tecnológicas, como Apple, Amazon o Ebay, apuestan por Texas para abrir nuevas sedes.

Como he dicho antes, nuestra intención es establecer una base sólida en Norteamérica y nuestra oficina en Dallas es una pieza fundamental en el puzle de nuestro negocio internacional.

\section{¿Qué recomendarían a la empresa española que mira a Texas como mercado para su expansión?}

Bueno, más que una recomendación, a las empresas españolas que miren a Texas como mercado para su expansión les daría un consejo, y es que tengan mucha paciencia, porque los inicios no son siempre tan rápidos como uno piensa cuando diseña un plan de negocio. Es fundamental al principio contar con una buena red de contactos y un respaldo legal, administrativo y contable solvente, porque de eso va a depender ser más ágil en los primeros pasos.

También es importante que sepan que deben contar con un buen riñón financiero, ya que la inversión es muy grande al principio y hay que establecer unas relaciones empresariales sólidas antes de recuperar toda la inversión en recursos.

Sin embargo, quiero dejar un mensaje positivo para las empresas españolas que quieran dar el salto a Estados Unidos, y es que el país y Texas, en particular, son un mercado muy seguro para hacer negocios: las reglas están muy bien definidas, las empresas son serias y comprometidas y la morosidad casi no existe, ya que las formas de pago están perfectamente definidas. Todo esto te permite tener un control y una estabilidad de las cuentas y del cash-flow de manera más fácil que en España.

\section{MAXAM}

\section{¿Cuál es la presencia de su empresa en Estados Unidos y, en particular, en Texas?}

La sede corporativa de MAXAM para todo su negocio en Estados Unidas está en la ciudad de Dallas. El Grupo MAXAM en Estados Unidos está presente a través de sus tres unidades de negocio:

1. MAXAM North America, cuyo negocio principal es la fabricación y venta de productos y soluciones de voladura para minería, canteras y obra civil.

2. RIO Ammunition, cuyo negocio principal es la fabricación y venta de cartuchos y componentes para la caza y tiro deportivo.

3. EXPAL USA, cuyo negocio principal son servicios de desmilitarización de munición proveniente del Ejército de Estados Unidos. 
Además de la sede corporativa en Dallas, MAXAM cuenta, también en Texas, con una fábrica de cartuchos en la ciudad de Marshall y otra fábrica de desmilitarización en Texarcana.

MAXAM comenzó a operar en América del Norte en el año 2001 mediante la compra de un operador local, Mining Services International (empresa dedicada a la fabricación de explosivos civiles y sistemas de iniciación que operaba en el continente desde 1979). Desde entonces, la compañía ha triplicado su tamaño, extendiendo su oferta de productos por todo el continente norteamericano.

\section{¿Por qué eligieron Texas como sede para el desarrollo de sus operaciones?}

Inicialmente la sede corporativa de MAXAM USA estaba ubicada en Salt Lake City (Utah). Por temas estratégicos y logísticos, se decidió en el año 2015 trasladar la sede a la ciudad de Dallas (Texas), con la finalidad de poder centralizar las tres unidades de negocio en un único punto, creando sinergias entre ellas.

\section{¿Qué ventajas ofrece Texas frente a otros Estados?}

Texas ofrece varias ventajas, pero destacamos su ubicación. La ubicación es clave, en el centro del país, de cara a la logística y transporte de nuestros productos, tanto dentro de Estados Unidos como por el resto del continente.

¿Cuáles son las mayores dificultades o retos a los que se ha enfrentado su empresa y en qué medida son particulares de este estado?

El reto que MAXAM ha encontrado es el alto nivel competitivo y la complejidad de los canales de distribución, especialmente a la hora de suministrar productos y servicios de voladuras. No obstante, en estas circunstancias, MAXAM ha sabido conjugar la oportunidad encontrando diversas fórmulas con la que proporcionar sus productos y servicios cumpliendo las expectativas de sus clientes.

Por otro lado, el mercado de los cartuchos de caza y tiro deportivo es muy maduro y, también, enormemente competitivo.

\section{¿Qué oportunidades ven en Texas a futuro?}

Texas es un estado cómodo y ágil para establecerse como negocio. Hay mucha oportunidad, especialmente bajo las actuales circunstancias económicas y del mercado laboral de EE UU.

¿Qué recomendarían a la empresa española que mira a Texas como un mercado para su expansión?

Cada empresa tiene su propia estrategia, tanto de implantación como de crecimiento. El mercado de EE UU es, en muchos sectores, el más grande del mundo. Las empresas españolas que decidan apostar por EE UU deben efectuar su propia due diligence, puesto que cada estado tiene su propia legislación e idiosincrasia, y parte del éxito del negocio es saber elegir bien dónde establecerse.

\section{T2O MEDIA}

\section{¿Cuál es la presencia de su empresa en Estados Unidos y, en particular, en Texas?}

T2O media, tras quince años en el mercado internacional, llega a EE UU a finales del año 2016. Texas es el lugar elegido como primera sede estadounidense, y desde ahí la agencia de medios opera para dar servicio a sus clientes con un enfoque que va en dos direcciones: ayudar a empresas españolas a dar el salto a este mercado y dar servicio a empresas locales que buscan mejorar sus resultados en EE UU y/o expandir su presencia al mercado europeo y latinoamericano. Tenemos un equipo de siete personas en Houston y un balance de extranjeros vs locales de 40/60, ya que consideramos que el conocimiento de la marca y el conocimiento del contexto deben ir de la mano: se trata de un mercado muy particular en muchos aspectos y es importante entenderlo para poder tener éxito.

\section{¿Por qué eligieron Texas como sede para el desarrollo de sus operaciones?}

Desde el inicio teníamos claro que buscábamos cubrir tres puntos clave para nuestro negocio: cercanía con el público y empresas hispanas (tanto de España como de Latinoamérica), un sitio en donde los beneficios $\square$ 
fiscales fueran atractivos tanto para la empresa, como para los trabajadores, y finalmente, un mercado con potencial de crecimiento y sin saturación, a nivel de marketing digital (como ocurre en el caso de los estados de Nueva York, Florida o California). Texas es uno de los estados que ofrece más beneficios fiscales, como es no tener state tax y, a su vez, tiene una diversidad multicultural muy atractiva para las empresas españolas y de diversas industrias, ya no solo del tradicional oil\&gas o medical (en los últimos años aparecen otras muy relevantes a nivel nacional, como manufacturas, tecnología, servicios, marítima, etcétera).

\section{¿Qué ventajas ofrece Texas frente a otros estados?}

La economía de Texas está valorada en 1,6 billones de dólares y en el último año ha incorporado más de 350.000 nuevos empleos; esto es: 1 de cada 7 empleos creados en EE UU (según publicaba CNBC en su estudio anual de 2018). Las diferencias por las que Texas es atractivo radican en su gran esfuerzo por ser un estado business-friendly, así como no tener un impuesto corporativo y ser sede de cerca de 52 empresas dentro del Rank de Forbes500. Otro factor que no se suele destacar, frente a otros estados, es el nivel de infraestructura que ofrece, contando con su importante puerto en Houston, junto con una serie de ciudades que están experimentando un gran crecimiento tecnológico, como puede ser el caso de Austin, en donde grandes corporaciones como Google, Facebook y Apple han apostado y han abierto nuevos centros de innovación y desarrollo, sin contar a grandes marca como DELL, AMD o 3M, que tienen presencia desde hace varios años. Otro de los principales motivos es que la tasa de desempleo del estado se mantiene cercana al $4 \%$, y para nosotros como agencia de medios digital representa una oportunidad muy importante en términos de negocio, crecimiento, ambiente laboral y, desde luego, oportunidades de entrada para empresas de EU y LATAM (ya que contamos desde hace más de ocho años con una oficina muy próxima en CDMX).

\section{¿Cuáles son las mayores dificultades o retos a los que se ha enfrentado su empresa y en qué medida son particulares de este estado?}

Se da por descontado que EE UU es un país con muchas diferencias, tanto culturales, como económicas y fiscales: cualquier empresa que busque establecerse en este mercado debe tenerlas en consideración antes de siquiera hacer su business plan. Pero nos parece que esto es un básico para cualquier mercado y negocio; lo que sí es una dificultad, que nadie te dice y no conoces hasta que llegas, es el hecho de que EE UU tiene una tasa de desempleo muy baja y en ciertas industrias los recursos humanos son muy complicados de gestionar, tanto a nivel de reclutamiento como respecto al nivel del trabajo desempeñado por cada empleado. Texas es un estado en el que ciertos perfiles son complicados de encontrar, lo que a veces puede llevar a tener que hacer reubicaciones, con lo que ello conlleva a nivel de costes para la empresa. El mercado estadounidense tiene sus peculiaridades en el momento en que comienzas a trabajar en él: es muy eficiente en muchas cosas, y a la vez extremadamente especializado en otras, lo que hace que en ocasiones las estructuras operativas tengan que ser mayores a las que tenemos en España, y con un nivel de costes bastante mayor. Por lo menos en la industria del marketing digital, en donde los salarios son bastante más elevados, pero no lo son siempre los costes del servicio que una agencia puede cobrar a un cliente, llegar a un break-even se hace más complejo al iniciar la operación, pues los resultados no se dan tan rápido como a veces nos gustaría. Por destacar un punto adicional, Texas ofrece oportunidades extraordinarias para empezar un negocio en EE UU, pero es importante cambiar la forma de pensar que a veces se tiene en Europa en cuanto a lo que tenemos y nuestras pretensiones. Es clave que entendamos primero cómo se hace negocio aquí, que el tema relacional es muy relevante y que los tiempos de maduración de una negociación son mucho más extensos respecto a lo que estamos acostumbrados. Si esto no lo tenemos en cuenta, podemos tener un burn-rate muy elevado y no previsto, lo que hace que, a los 18 meses de vida, muchos negocios que dan el salto estén, desafortunadamente, de vuelta. En el caso de T2O Media, ya hemos pasado este hito y estamos orgullosos de seguir creciendo y ofreciendo a nuestros clientes, tanto locales como españoles, un servicio de gran calidad, con una cercanía que permite relacionarnos con EE UU y Europa de forma más consistente. 


\section{¿Qué oportunidades ven en Texas a futuro?}

Texas y, más en concreto, Houston tienen la ventaja y desventaja de depender mucho del precio del barril de petróleo. Este ha subido en los últimos dos años, y recuperó algo de sus niveles normales, aunque a finales de 2018 ha vuelto a descender un poco, para cerrar cerca de los 60 dólares por barril. No podemos tampoco olvidar a Harvey, que trajo consigo muchos problemas e historias muy tristes, pero sirvió para reactivar el sector de los servicios y la construcción durante 2018, y de hecho se prevé que continúe así a lo largo de 2019. Resaltamos esto porque es un indicador financiero que impacta sustancialmente, cómo esta ciudad funciona y evoluciona, algo que se puede entender de forma más clara en un artículo publicado por la Universidad de Bauer en Houston (https://www.bauer.uh.edu/centers/irf/houston-updates.php). Dicho esto, T2O media decidió asentarse en Texas por ser una apuesta a largo plazo, en donde el estado continuará en crecimiento, las grandes empresas seguirán invirtiendo y la relación americano-hispana, bajo un enfoque multicultural, hará que sea un mercado muy atractivo para atraer inversiones de otros países de Europa y Latinoamérica. Vemos como una oportunidad no entrar en mercados altamente competitivos dentro de nuestro entorno digital, como pueden ser los ya citados de Nueva York y Miami, y sobre todo poder ofrecer un servicio que brinde a nuestros clientes un valor añadido real en términos de conocimiento del mercado.

Nos hicimos la pregunta de qué recomendar a empresas españolas antes de venir, y ahora, después de dos años en Houston, podemos resumir en tres puntos principales lo que a nuestro parecer es indispensable para el éxito de un negocio español que busca dar este salto:

- Trabajar el plan de negocio de la mano de una consultora local, que pueda asesorar de forma más localizada cuál es la mejor forma de hacer este desembarco e ir de la mano de ICEX para este proceso, ya que ofrecen diversas ventajas y planes de ayuda para hacer este proceso más sólido y bien planificado.

- Pensar qué es lo que busca EE UU de estas nuevas empresas que aterrizan en su territorio: generación de empleo, servicios de calidad y diferenciación contundente. Si estos tres puntos se dan y se trabaja para demostrar que no se viene a quitar trabajo a la fuerza laboral americana, sino a potenciarla y ayudarla a crecer, el mercado, y más en concreto Texas, responde de manera excepcional.

- Finalmente, tener controlado todo lo referente a temas de inmigración y visados, cumplir con las normas de TWC (comisión de trabajo de Texas) y las regulaciones locales a nivel empleados, seguros y fiscalidad (es crítico para evitar tener problemas a medio plazo). A veces pensamos que las cosas se hacen igual que en España, pero en realidad son muy distintas, y los procesos, formas de comunicarse y tiempos cambian drásticamente.

En conclusión, tener los objetivos claros, no tener miedo a invertir y arriesgar, y ser consistentes en el proceso son elementos clave para que un negocio español tenga éxito en estas latitudes. 